\title{
A MATHEMATICAL MODEL FOR THE DETERMINATION OF VISCOELASTIC BEHAVIOR OF BRAIN IN VIVO-I OSCILLATORY RESPONSE*
}

\author{
HAN CHOU WANG and ALAN S. WINEMAN ${ }^{\dagger}$ \\ Highway Safety Research Institute. The University of Michigan. Ann Arbor, Michigan \\ 48104 , U.S.A.
}

\begin{abstract}
In a recent experiment for determining the mechanical response of brain in vivo, a probe, inserted through scalp, skull and dura, is placed in contact with and normal to the brain. given a prescribed motion, and the time variation of corresponding force is measured. In the corresponding continuum mechanical model, brain is idealized as a linear isotropic viscoelastic solid constrained by a rigid skull. At the mating surface, the shear stress and normal displacement vanish everywhere except under the probe which exerts a local radial displacement. This model introduces effective viscoelastic moduli in shear, which is unknown, and in dilation, which is considered known from other sources. Part l of this study considers steady oscillations of the probe. A transcendental equation for the complex shear modulus is established in terms of probe displacement and force amplitude ratio and phase lag and is solved for specific test data. The corresponding stress and displacement fields are evaluated so that the probe influence may be assessed.
\end{abstract}

\section{INTRODUCTION}

IN RECENT years, there has been a great deal of interest in the study of mechanical phenomena associated with head injury, with particular interest in mechanisms of brain damage. A major restraint in the study has been the lack of knowledge of the mechanical properties of brain tissue. It thus becomes necessary to determine which constitutive assumptions apply to brain tissue and then to measure the material parameters defined by these assumptions. Existing theoretical analyses of brain damage mechanisms, discussed by Engin (1969), model brain as an inviscid compressible fluid. Although this model may be adequate for studying brain damage due to compressive waves. past experimental work on brain tissue from a variety of species, discussed by Fallenstein, Hulce and Melvin (1969), suggests that brain exhibits viscoelastic properties. Most of this experimental work, however, suffers from having been done in vitro. Robbins and Roberts (1967) have indicated that deterioration of brain tissue after death is so fast that experiments on brain tissue must be conducted as soon as possible after the time of death. For this reason, there exists the need for experiments conducted in vivo which measure the mechanical properties of brain tissue.

From a biomechanics point of view, it is quite natural to expect that most biological materials would display both elastic or solid like properties and viscous or dissipative properties. This is because these materials must continually absorb and damp out all kinds of transient loads. This is further reinforced by Jamison's observation (1968) that when compared to engineering materials, brain can be likened to a soft gel. In tensile or compressive oscillatory tests on cylindrical brain samples, Galford and McElhaney (1970) showed that force and displacement were out of phase. Since in an elastic solid, these would

\footnotetext{
*Received 24 February 1972.

†Associate professor. Department of Engineering Mechanics.
} 
be in phase and in a viscous fluid they would be $90^{\circ}$ out of phase, these tests further suggests that brain is viscoelastic. They also showed that brain exhibits viscoelastic properties of creep and relaxation.

In addition to the assumption of viscoelasticity, brain has been modeled as a linear, isotropic and homogeneous material. The stress-strain law can then be characterized by two independent material parameters, such as a tensile, shear or bulk modulus, or a viscoelastic analog of Poisson's ratio. These will be independent of stress or strain level and depend only on time or frequency as in the case of an oscillatory test. The assumption of linearity has not been conclusively verified experimentally, one way or the other. Isotropy seems not to have been discussed in the literature. Although brain is clearly not homogeneous, this assumption at least reduces the problem to one of finding two material parameters which are functions of at most one variable. These parameters' are then 'average' in a sense defined by the experimental situation used to determine them. Holbourn (1943) proposed that brain injury is due to shear deformation. Thus, the pertinent material parameters would be the shear modulus governing the distortional response and the bulk modulus, governing the dilatational response.

Experiments conducted in vivo can be devised to measure such quantities indirectly. Time dependent material parameters can be determined by means of experiments involving wave propagation. However, for complex geometries, these are difficult to analyze. Creep or relaxation experiments can be conducted assuming quasi-static conditions, but these give very little useful information about short time response when inertial effects are still important. For this reason, interest centers on the material response and properties arising in experiments involving sinusoidal oscillations. Such tests give bulk and shear moduli as complex functions of frequency. If these moduli are known over a wide range of frequencies, then their time dependent equivalents can be obtained by the procedure outlined by Gross (1957) and Ferry (1956).

Recently Fallenstein et al. (1969) developed an experiment to be performed directly on a living Rhesus monkey's brain. Since the present investigation is a theoretical analysis of Fallenstein's experiment, a more detailed description of this operation is stated as follows. A monkey was anesthetized with sodium pentobarbital and placed in a jig designed to hold the head firmly in the upright position. A t-in. hole was cut through the skull directly over the right frontal lobe and close to the midline. The dura was then removed with a scalpel. A small dynamic probe with $0.1 \mathrm{~cm}^{2}$ area was positioned vertically over the hole. The probe was attached to an impedance head given an initial presence and then vibrated perpendicular to and in contact with the surface of the brain. Thus, the force, displacement and phase angle between force and displacement were measured from experiment. In order to analyze the experimental results, a theoretical model is assumed as shown in Fig. 1. In this theoretical model, the skull is assumed to be a rigid spherical shell since the skull is much stiffer than brain tissue. The brain, assumed to be a linear viscoelastic material, fills the interior. The experiment is interpreted as the mechanics problem of finding the steady state response characteristics of a solid sphere of linear viscoelastic material bounded by a rigid spherical container and subjected to a local radial harmonic excitation. In particular, an equation is established for the material parameters in terms of the force and displacement of the excitation. A technique is developed for solving for these parameters when experimental data is known.

In addition to determining material parameters in an oscillatory test, it is of interest to estimate the order of magnitude of relaxation or creep moduli and a typical relaxation or creep time of brain tissue. Fallenstein's probe test can be modified for this purpose. In the relaxation test the probe is subjected to an 


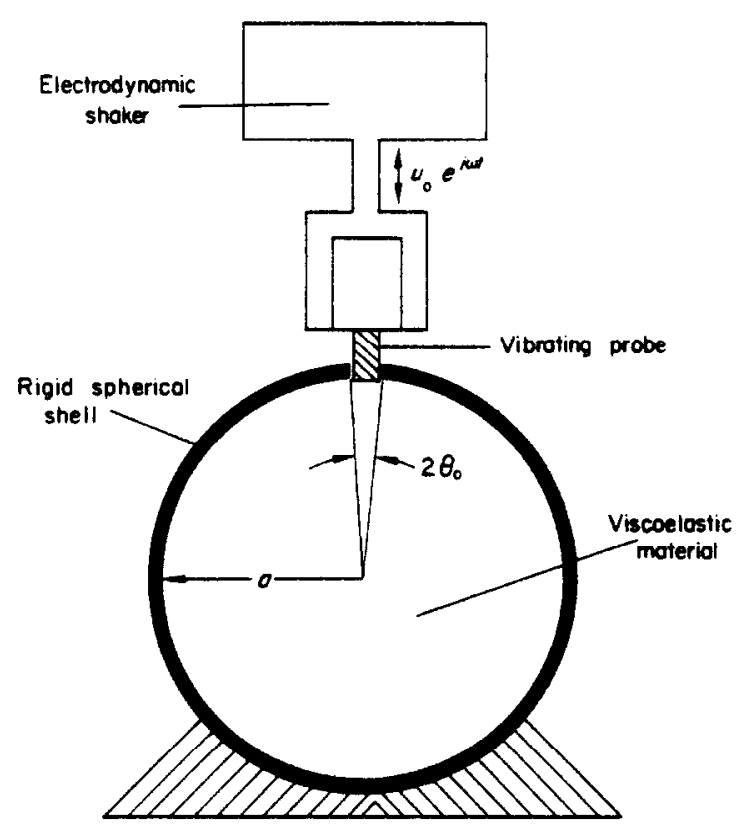

Fig. 1. Model for theoretical analysis.

instantaneously applied displacement which is held constant while the time decay of force is measured. In the creep test, a constant force is applied to the probe and the time increasing displacement is measured. The assumptions used in the theoretical model for the oscillatory test are the same here except for the time dependent form of the boundary condition giving the radial displacement. An equation between time dependent material moduli and experimental data can again be established. This will be discussed further in part II of the present study.

Recently, Engin and Wang (1970) considered the same physical problem as discussed here, but used a different approach. First, they treated their problem as an elastic problem to which Bland's (1960) so-called correspondence principle was applied to get the viscoelastic response. In the present investigation, the problem is formulated directly from viscoelasticity theory. This unifies the treatments of the oscillatory and creep or relaxation probe tests. Secondly, they consider the brain as an incompressible visco- elastic material. Here the bulk modulus is assumed to be a constant whose value is the same as that for fresh water (Goldsmith, 1966). Thirdly, the probe profile has been changed to allow a smoother surface displacement. Fourth, Engin and Wang did not determine the shear modulus for specific experimental data, which is done in the present work. Finally. using this value of the shear modulus, the stress and displacement distribution in the sphere are presented so that the influence of the probe can be evaluated.

The basic and general theory of linear viscoelasticity can be found from the books of Gross (1953), Bland (1960) and Flugge (1967). A review of the basic constitutive equation for a linear isotropic, viscoelastic material undergoing sinusoidal oscillations is presented in Section 2. In Section 3, the idealization of the experiment into its mathematical model is carried out, giving the governing equations and boundary conditions. $A$ representation for the solution to the boundary value problem is derived in Section 4. Expressions for stresses and displacements are obtained in the form of infinite series involving Legendre polynomials and spherical Bessel's function. Section 5 first treats the numerical issues which arise in computing these series. Then a numerical and graphical method for finding the complex shear modulus, $\mu^{*}$, is outlined. The stress and displacement distributions in the sphere calculated for the experimentally determined value of the shear modulus are presented and discussed in Section 5.

\section{CONSTITUTIVE EQUATION}

The general three dimensional form of the constitutive equation for isotropic linear viscoelastic materials was developed in the treatise by Gurtin and Sternberg (1962). It was shown that this model depends on two independent material parameters. These can be taken to represent the response in pure shear and in hydrostatic loading. The present work is concerned with the response of linear viscoelastic materials undergoing sinusoidal oscillations. 
In this case the tensorial strain history has the form $\epsilon_{i j}(t)=\epsilon_{i j} e^{i \omega t}$, where $\epsilon_{j}$ is independent of time. Substituting this strain history into the general three-dimensional constitutive equation gives a tensorial stress history of form $\sigma_{i j}(t)=\sigma_{i j} e^{i \omega t}$, where $\sigma_{i j}$ is independent of time and related to $\epsilon_{i j}$ by

$\sigma_{i j}=2 \mu^{*}(i \omega) \epsilon_{i j}+\frac{1}{3} \delta_{i j}\left[3 k^{*}(i \omega)-2 \mu^{*}(i \omega)\right] \epsilon_{k k}$

$\mu^{*}(i \omega)$ and $k^{*}(i \omega)$ are complex valued functions of frequency $\omega$ and are called the complex moduli in shear and bulk response, respectively. The relation of these material parameters to their time dependent analogs is discussed in the book by Gross (1953).

\section{FORMULATION OR THE CONTINUUM MODEL, GOVERNING EQUATIONS}

The basic details of Fallenstein's probe test were presented in the Introduction. Before formulating the model in any more detail, it is useful to consider more carefully the nature of the loading on the brain.

After the probe tip was positioned on the exposed cerebral cortex, the probe was pressed into the brain surface through a screw drive mechanism, the static brain deformation being measured with an attached dial indicator. While at a specified static deformation, the probe was driven with a small sinusoidal amplitude. Then the transferred force and dynamic displacement signals from the probe were displayed on a dual-beam oscilloscope and also recorded on magnetic tape. From the oscilloscope displays, the amplitude of displacement and force and its phase lag between force and displacement were determined. Some of the experimental results obtained aret:

1. Test No. 5/2-20-69

Frequency $=80 \mathrm{~Hz}$.

Static Deformation $=0.348 \mathrm{~cm}$

Double Amplitude Force $=1 \cdot 155 \times 1 \dot{u}^{2}$ dynes
Double Amplitude Displacement $=8 \cdot 59$ $\times 10^{-3} \mathrm{~cm}$

Phase $\mathrm{Lag}=\tan ^{-1} 0.665$

2. Test No. 2-A/2-20-69

Frequency $=96 \mathrm{~Hz}$.

Static Deformation $=0.183 \mathrm{~cm}$

Double Amplitude Force $=9.02 \times 10^{3}$ dynes

Double Amplitude Displacement $=4 \cdot 7$ $\times 10^{-2} \mathrm{~cm}$

Phase Lag $=\tan ^{-1} 0.55$

3. Test No. 6/2-20-69

Frequency $=80 \mathrm{~Hz}$.

Static Deformation $=0.273 \mathrm{~cm}$

Double Amplitude Force $=10.86 \times 10^{3}$ dyn

Double Amplitude Displacement $=3.6$ $\times 10^{-2} \mathrm{~cm}$

Phase Lag $=\tan ^{-1} 0.604$.

Comparing the above test results, it is seen that the response is non-linear. Possible sources of non-linearity might be either material non-linearity or a large initial preset (or static deformation). In order to avoid the effects of material non-linearity, test data of the smallest superposed displacement will be used in later computation, i.e. test data No. 1 above. Because of the small amount of data and the possibility of geometric non-linearity induced by the large preset, this data will only be used to show the procedure for finding the complex shear modulus when better test results are available. Besides the information measured from Fallenstein's experiment, the bulk modulus can be obtained from other sources. According to Goldsmith (1966), the bulk modulus of brain is similar to water, a constant whose value is $3 \times 10^{5}$ psi. The bulk modulus appears in the expression used to find the complex shear modulus or relaxation function of brain tissue.

General assumptions of the continuum model

An initial idealization of Fallenstein's probe test from the mechanics point of view was 
given in the Introduction. The local radial excitation is treated as a local displacement which is imposed directly and perpendicular to the surface of the soft material. The displacement consists of a small fixed preset upon which is superposed a local harmonic oscillation. The complete list of assumptions used in constructing this model are summarized as follows:

1. Brain tissue will be assumed as a linear, homogeneous, isotropic viscoelastic material.

2. The brain entirely fills the skull which is assumed as a spherical case.

3. Since the skull is much harder than the brain tissue, a rigid material for the skull is assumed.

4. The preset is so small that the displacement fields due to preset and to oscillation can be superposed.

5. The excitation load is axisymmetric, which implies the response will also be axisymmetric about an axis through the line of force of the excitation load. Also, displacements will occur only in planes through the line of force.

6. The connection between the brain and the skull is complex. Generally, there is a thin layer of cerebrospinal fluid between the skull and brain. In addition, there are nerve bundles and blood vessels at the lower part of the brain. Since the probe is placed in a section of the skull far from the restraint of nerve cords, the influence of this is neglected. Furthermore, since the layer of cerebrospinal fluid is thin and its viscosity is small (similar to the water), the additional assumption is made that the shear stress at the interface between brain and skull can be neglected. This should be a good approximation at least at low frequencies.

7. Body force is neglected.

Based on the above assumptions, a theoretical model is pictured as shown in Fig. 1. We introduce spherical coordinates $(r, \theta, \varphi)$ with origin at the center of the sphere and with the center line of the probe lying on $\theta=0$.

\section{Continuum model-governing equations}

Assumption 6 means that there is no shear stress at the interface of viscoelastic material and rigid container. Furthermore, assumption 5 implies that $\sigma_{r} \equiv 0$. The stress boundary condition at $r=a$ reduces to

$$
\sigma_{r \theta}(a, \theta, t)=0
$$

Although there is no basis for specifying a boundary condition on the normal component of stress, the model suggests a boundary condition on the normal component of displacement, $u_{r}$. Since the skull is assumed rigid, the normal displacement must vanish everywhere except at the opening provided by the probe. Under the probe the displacement is prescribed. The boundary condition on normal displacement $u_{r}(a, \theta, t)$ then becomes

$$
\begin{aligned}
& u_{r}(a, \theta, t)=u_{r}(\theta, t) \\
& = \begin{cases}u_{p}(\theta)+u(\theta) e^{i \omega t} & 0 \leqslant \theta \leqslant \theta_{0} \\
0 & \theta_{0} \leqslant \theta \leqslant \pi\end{cases}
\end{aligned}
$$

where $u_{p}(\theta)$ is the displacement due to the preset. The normal displacement $u_{r}(a, \theta, t)$ will have its maximum value under the probe, or at $\theta=0$, then decrease gradually as $\theta$ increases and finally reach zero at the angle $\theta=\theta_{0}$. For simplicity, $u(\theta)$ is chosen as

$$
u(\theta)=\frac{u_{0}}{2}\left(1+\cos \frac{\pi \theta}{\theta_{0}}\right) \quad 0 \leqslant \theta \leqslant \theta_{0}
$$

where $u_{0}$ is the maximum amplitude of vibrating probe. (See Fig. 2.)

Letting $\rho$ be the mass density of the medium, the equation of motion for small deformations, with respect to a cartesian coordinate system, is

$$
\frac{\partial \sigma_{i j}(\mathbf{X}, t)}{\partial x_{j}}=\rho \frac{\partial^{2} u_{i}(\mathbf{X}, t)}{\partial t^{2}}
$$

If both the preset and oscillation deformations are small, the field equations can be considered linear. Boundary conditions are aiso linear and equation (2) can apply to both preset and oscillation solutions. Also, the 


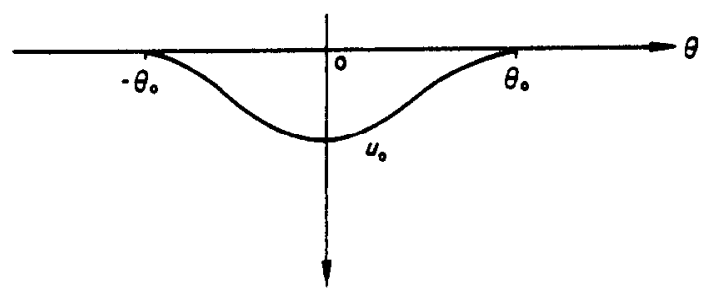

Fig. 2. The local radial displacement on the surface.

preset state is static and does not generate an inertial term in equation ( 5 ). For these reasons, the preset problem and oscillation part of the motion superpose and can be treated independently. It is assumed that the probe is calibrated to read zero force in the preset state as was done in the experiment. Without loss in generality, one can then assume $u_{p}=0$. Since the applied load (displacement) is sinusoidal, the displacement vector can be written as

$$
\mathbf{u}(\mathbf{X}, t)=\mathbf{u} e^{i \omega t}
$$

which, assuming small deformations, implies the strains are sinusoidal. With respect to spherical coordinates and utilizing assumption 5 , the strain-displacement equations reduce to

$$
\begin{aligned}
& \epsilon_{r r}=\frac{\partial u_{r}}{\partial_{r}} \\
& \epsilon_{r \theta}=\frac{1}{2}\left(\frac{1}{r} \frac{\partial u_{r}}{\partial \theta}+\frac{\partial u_{\theta}}{\partial r}-\frac{u_{\theta}}{r}\right) \\
& \epsilon_{\theta \theta}=\frac{u_{r}}{r}+\frac{1}{r} \frac{\partial u_{\theta}}{\partial \theta} \\
& \epsilon_{\varphi \varphi}=\frac{u_{r}}{r}+\frac{u_{\theta}}{r} \cot \theta .
\end{aligned}
$$

Substituting $\sigma_{i j}(\mathbf{X}, t)=\sigma_{i j}(\mathbf{X}) e^{i \omega t}$ and equation (6) into equation (5) and using equation (1), the equation of motion becomes

$$
\begin{array}{r}
\mu^{*}(i \omega) \nabla^{2} \mathbf{u}+\left[\lambda^{*}(i \omega)+\mu^{*}(i \omega)\right] \nabla(\nabla \cdot \mathbf{u}) \\
=-\rho \omega^{2} \mathbf{u}
\end{array}
$$

where $\lambda^{*}=\left(3 k^{*}-2 \mu^{*}\right) / 3$ is called the complex Lamé modulus.

\section{SOLUTION}

The displacement vector $u$ in equation (8) can be represented in terms of displacement potentials in the form [Love, 1944]

$$
\mathbf{u}=\nabla \Phi+\nabla \times \psi
$$

where $\Phi$ is a scalar function and $\psi$ is a vector function. It can be shown (Sokolnikoff, 1956) that when the displacement vector $\mathbf{u}$ has this representation, equation (8) is satisfied if

$$
\begin{aligned}
& \nabla^{2} \Phi=-k_{1}{ }^{2} \Phi, k_{1}{ }^{2}=\rho \omega^{2} /\left(\lambda^{*}+2 \mu^{*}\right) \\
& \nabla^{2} \psi=-k_{2}{ }^{2} \psi, k_{2}{ }^{2}=\rho \omega^{2} / \mu^{*} .
\end{aligned}
$$

Both of these equations are the standard form of wave equation, which can be solved by the separation of variable method when expressed in terms of spherical coordinates $(r, \theta, \varphi)$. Since the problem is axisymmetric, $\Phi$ and $\psi$ are functions of $r$ and $\theta$ only. The condition based on assumption $S$ that $u_{\varphi}=0$ implies that $\Psi_{r}=\Psi_{\theta}=0, \Psi_{\varphi}=\Psi(r, \theta) \neq 0$. When written with respect to spherical coordinates, equation (10) reduces to

$\frac{\partial^{2} \Phi}{\partial r^{2}}+\frac{2}{r} \frac{\partial \Phi}{\partial r}+\frac{1}{r^{2}} \frac{\partial^{2} \Phi}{\partial \theta^{2}}+\frac{\cos \theta}{r^{2} \sin \theta} \frac{\partial \Phi}{\partial \theta}=-k_{1}^{2} \Phi$,

and equation (11) becomes

$$
\begin{aligned}
\frac{\partial^{2} \Psi}{\partial r^{2}}+\frac{2}{r} \frac{\partial \Psi}{\partial r}+\frac{1}{r^{2}} \frac{\partial^{2} \Psi}{\partial \theta^{2}}+\frac{\cos \theta}{r^{2} \sin \theta} & \frac{\partial \Psi}{\partial \theta}-\frac{\Psi}{r^{2} \sin ^{2} \theta} \\
& =-k_{2}^{2} \Psi .
\end{aligned}
$$

Assuming separation of variables representations for $\Phi$ and $\Psi$, the following expressions are obtained as solutions to equations (12-13):

$$
\Phi=\sum_{n=0}^{\infty} a_{n} j_{n}\left(k_{1} r\right) P_{n}(\cos \theta)
$$

and

$$
\Psi=\sum_{n=1}^{\infty} b_{n} j_{n}\left(k_{2} r\right) P_{n}^{1}(\cos \theta)
$$


where $P_{n}(\cos \theta), \quad(n=0,1,2, \ldots), \quad$ are Legendre polynomials, $P_{n}^{1}(\cos \theta)$ are associated Legendre functions of order $1, j_{n}(z)$, $(n=0,1,2, \ldots)$ are spherical Bessel functions of the first kind, and $a_{n}, b_{n}$ are constants to be determined.

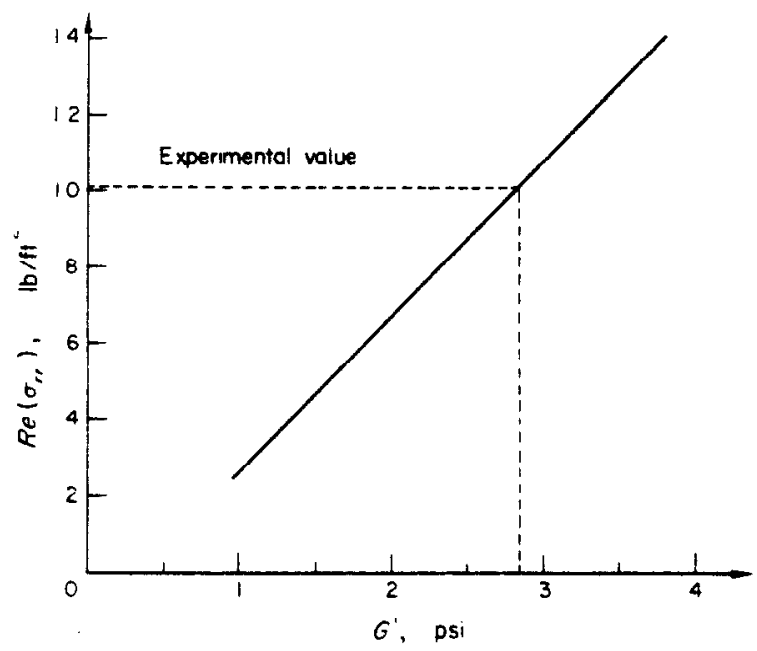

Fig. 3. Real part of normal stress vs. real part of complex shear modulus.

Equations (14) and (15), together with equation (9), represent the general solution to the governing equation (8). The undetermined constants $a_{n}, b_{n}$ are to be found by applying the boundary conditions on stress and displacement of equations (2) and (4). Before this can be done, it is convenient to calculate u. Substituting equations (14) and (15) into equation (9), one obtains

$$
\begin{aligned}
\mathbf{u}= & \sum_{n=0}^{\infty}\left[a_{n} k_{1} j_{n}^{\prime}\left(k_{1} r\right)+\frac{b_{n} n(n+1)}{r}\right. \\
& \left.\times j_{n}\left(k_{2} r\right)\right] P_{n}(\cos \theta) \mathbf{e}_{r} \\
& -\frac{1}{r} \sum_{n=1}^{\infty}\left[a_{n} j_{n}\left(k_{1} r\right)+b_{n} j_{n}\left(k_{2} r\right)\right. \\
& \left.+b_{n} k_{2} r j_{n}^{\prime}\left(k_{2} r\right)\right] P_{n}{ }^{1}(\cos \theta) \mathbf{e}_{\theta} \\
= & u_{r} \mathbf{e}_{r}+u_{\theta} \mathbf{e}_{\theta}
\end{aligned}
$$

where ( $)^{\prime}$ denotes differentiation of a function with respect to its argument and where $u_{r}$ and $u_{\theta}$ are the radial and tangential displacements, respectively.

The first boundary condition, equation (2), implies by equation (1) that $\epsilon_{r \theta}(a, \theta)=0$. Combining the expressions for displacement components $u_{r}$ and $u_{\theta}$ implied by equation (16) in this condition and equation (7) and then using the linear independence of the associate Legendre functions gives one relationship between $a_{n}$ and $b_{n}$.

A second relationship follows from the displacement boundary condition, equation (3), where now $u_{p}=0$. The function $u(\theta)$ in equation (4) can be expanded in a series of Legendre polynomials.

$$
u(\theta)=\sum_{n=0}^{\infty} c_{n} P_{n}(\cos \theta)
$$

Substituting from equation (16) into equation (4), using the linear independence of Legendre polynomials and the preceding results gives $a_{n}$ and $b_{n}$ in terms of $c_{n}$.

The series representing stress and displacements obtained by this procedure contain the first and second derivatives of the spherical Bessel functions and can be found elsewhere (Wang, 1971). In order to put them in a form suitable for numerical computation, the series were first expressed in terms of spherical Bessel functions using well-known recurrence relations (Handbook of Mathematical Functions, 1964).

The final form of the series representing the stress and displacement components becomes

$$
\begin{aligned}
\sigma_{r r}= & \frac{1}{r^{2}} \sum_{n=0}^{\infty} a_{n}\left\{\left[-\left(\lambda^{*}+2 \mu^{*}\right) k_{1}{ }^{2} r^{2}\right.\right. \\
& \left.\left.+2 \mu^{*}\left(n^{2}-n\right)\right] j_{n}\left(k_{1} r\right)+4 \mu^{*} k_{1} r j_{n+1}\left(k_{1} r\right)\right\} \\
& \times P_{n}(\cos \theta)+\frac{2 \mu^{*}}{r^{2}} \sum_{n=1}^{\infty} \lambda_{n} b_{n}\left[(n-1) j_{n}\left(k_{2} r\right)\right. \\
& \left.-k_{2} r j_{n+1}\left(k_{2} r\right)\right] P_{n}(\cos \theta)
\end{aligned}
$$




$$
\begin{aligned}
\sigma_{r \theta}= & \frac{\mu^{*}}{r^{2}} \sum_{n=1}^{\infty} 2 a_{n}\left[(1-n) j_{n}\left(k_{1} r\right)+k_{1} r j_{n+1}\left(k_{1} r\right)\right] \\
& \times P_{n}{ }^{1}(\cos \theta)+\frac{\mu^{*}}{r^{2}} \sum_{n=1}^{\infty} b_{n}\left[\left(2-2 n^{2}\right.\right. \\
& \left.\left.+k_{2}{ }^{2} r^{2}\right) j_{n}\left(k_{2} r\right)-2 k_{2} r j_{n+1}\left(k_{2} r\right)\right] P_{n}{ }^{1}(\cos \theta)
\end{aligned}
$$$$
\sigma_{\theta \theta}=\frac{2 \mu^{*}}{r^{2}} \sum_{n=0}^{\infty} a_{n}\left[\left(-n^{2}-\frac{\lambda^{*} k_{1}{ }^{2} r^{2}}{2 \mu^{*}}\right) j_{n}\left(k_{1} r\right)\right.
$$$$
\left.-k_{1} r j_{n+1}\left(k_{1} r\right)\right] P_{n}(\cos \theta)+\frac{2 \mu^{*}}{r^{2}} \cot \theta \sum_{n=1}^{\infty}
$$$$
\times a_{n} j_{n}\left(k_{1} r\right) P_{n}{ }^{1}(\cos \theta)-\frac{2 \mu^{*}}{r^{2}} \sum_{n=1}^{\infty} \lambda_{n} b_{n}\left[n j_{n}\left(k_{2} r\right)\right.
$$$$
\left.-k_{2} r j_{n+1}\left(k_{2} r\right)\right] P_{n}(\cos \theta)+\frac{2 \mu^{*}}{r^{2}} \cot \theta \sum_{n=1}^{\infty}
$$$$
\times b_{n}\left[(n+1) j_{n}\left(k_{2} r\right)-k_{2} r j_{n+1}\left(k_{2} r\right)\right] P_{n}{ }^{1}(\cos \theta)
$$$$
\sigma_{\varphi \varphi}=\frac{1}{r^{2}} \sum_{n=0}^{\infty} a_{n}\left[\left(2 \mu^{*} n-\lambda^{*} k_{1}{ }^{2} r^{2}\right) j_{n}\left(k_{1} r\right)\right.
$$$$
\left.-2 \mu^{*} k_{1} r j_{n+1}\left(k_{1} r\right)\right] P_{n}(\cos \theta)-\frac{2 \mu^{*}}{r^{2}} \cot \theta \sum_{n=1}^{\infty}
$$$$
\times a_{n} j_{n}\left(k_{1} r\right) P_{n}^{1}(\cos \theta)+\frac{2 \mu^{*}}{r^{2}} \sum_{n=1}^{\infty} \lambda_{n} b_{n} j_{n}\left(k_{2} r\right)
$$$$
\times P_{n}(\cos \theta)-\frac{2 \mu^{*}}{r^{2}} \cot \theta \sum_{n=1}^{\infty} b_{n}\left[(1+n) j_{n}\left(k_{2} r\right)\right.
$$$$
\left.-k_{2} r j_{n+1}\left(k_{2} r\right)\right] P_{n}^{1}(\cos \theta)
$$$$
u_{r}=\frac{1}{r} \sum_{n=0}^{\infty}\left\{a_{n}\left[n j_{n}\left(k_{1} r\right)-k_{1} r j_{n+1}\left(k_{1} r\right)\right]\right.
$$$$
\left.+\lambda_{n} b_{n} j_{n}\left(k_{2} r\right)\right\} P_{n}(\cos \theta)
$$$$
u_{\theta}=-\frac{1}{r} \sum_{n=1}^{\infty}\left\{a_{n} j_{n}\left(k_{1} r\right)+b_{n}\left[(n+1) j_{n+1}\left(k_{2} r\right)\right.\right.
$$$$
\left.\left.-k_{2} r j_{n+1}\left(k_{2} r\right)\right]\right\} P_{n}^{1}(\cos \theta) .
$$

Also, constants $a_{n}$ and $b_{n}$ can be rewritten as

$$
\begin{aligned}
& a_{n}=\frac{a \cdot f_{1}(n)}{f_{3}(n) \cdot f_{1}(n)+2 \lambda_{n} \cdot f_{2}(n) j_{n}\left(k_{2} a\right)} \cdot c_{n} \\
& b_{n}=\frac{2 a \cdot f_{2}(n)}{f_{3}(n) \cdot f_{1}(n)+2 \lambda_{n} \cdot f_{2}(n) \cdot j_{n}\left(k_{2} a\right)} \cdot c_{n}
\end{aligned}
$$

where

$$
\begin{aligned}
f_{1}(n) & =\left(2 n^{2}-2-k_{2}^{2} a\right) j_{n}\left(k_{2} a\right)+2 k_{2} a j_{n+1}\left(k_{2} a\right) \\
f_{2}(n) & =(1-n) j_{n}\left(k_{1} a\right)+k_{1} a j_{n+1}\left(k_{1} a\right) \\
f_{3}(n) & =n j_{n}\left(k_{1} a\right)-k_{1} a j_{n+1}\left(k_{1} a\right) \\
\lambda_{n} & =n(n+1) .
\end{aligned}
$$

\section{NUMERICAL RESULT AND DISCUSSION}

The general expressions for the stress and displacement components which were derived in Section 3 are in the form of infinite series involving Legendre polynomials, associated Legendre polynomials and spherical Bessel functions. In the present section, we will first describe the method used to compute these series. and then outline the method for determining the complex shear modulus corresponding to given measured input and response data.

\section{Computation of series}

The computation of Legendre polynomials $P_{n}(\cos \theta)$ and associated Legendre polynomials $P_{n}{ }^{1}(\cos \theta)$ was carried out using standard recurrence relations (Handbook of Mathematical Functions, 1964) and presented no difficulties.

The following series representation is used to calculate the spherical Bessel function (Handbook of Mathematical Functions, 1964)

$$
\begin{aligned}
& j_{n}(z)=\frac{z^{n}}{1 \cdot 3 \cdot 5 \cdots(2 n+1)}\left[1-\frac{z^{2} / 2}{1 !(2 n+3)}\right. \\
& +\frac{\left(z^{2} / 2\right)^{2}}{2 !(2 n+3)(2 n+5)}-\cdots \\
& \left.+\frac{(-1)^{k}\left(z^{2} / 2\right)^{k}}{k !(2 n+3)(2 n+5) \cdots(2 n+2 k+1)}+\cdots\right]
\end{aligned}
$$


The series converges for all $z$. For each fixed $z$, the series inside the brackets tends to 1 as $n \rightarrow \infty$ while the coefficient of the brackets approaches zero. Hence $j_{n}(z)$ also approaches zero as $n$ approaches to infinity for each fixed $z$. From equation (19), it is easily seen that the numerators of $a_{n}$ and $b_{n}$ are linear in $j_{n}(z)$ while their denominators are quadratic. Thus, as $n$ approaches infinity, these coefficients become unbounded. However, $a_{n}$ and $b_{n}$ appear in the series in the combination of $a_{n} j_{n}\left(k_{1} r\right), a_{n} j_{n+1}\left(k_{1} r\right), b_{n} j_{n}\left(k_{2} r\right)$ and $b_{n} j_{n+1}\left(k_{2} r\right)$ which converge as $n$ becomes large. If the factors in those products are calculated separately. numerical inaccuracies arise and the series for, say, $\sigma_{r r}$ does not approach a limit. This problem is avoided by calculating the products of $a_{n} j_{n}\left(k_{1} r\right), a_{n} j_{n+1}\left(k_{1} r\right), b_{n} j_{n}\left(k_{2} r\right)$ and $b_{n} j_{n+1}\left(k_{2} r\right)$ after they have been simplified.

For example, if we divide both numerator and denominator in the expression

$$
a_{n} j_{n}\left(k_{1} r\right)=\frac{a f_{1}(n) \cdot j_{n}\left(k_{1} r\right)}{f_{3}(n) f_{1}(n)+2 \lambda_{n} f_{2}(n) j_{n}\left(k_{2} a\right)} \cdot c_{n}
$$

by $j_{n}\left(k_{1} a\right) \cdot j_{n}\left(k_{2} a\right)$, we obtain

$$
a_{n} j_{n}\left(k_{1} r\right)=\frac{a F_{1}(n) \cdot j_{n}\left(k_{1} r\right) / j_{n}\left(k_{1} a\right)}{F_{3}(n) \cdot F_{1}(n)+2 \lambda_{n} F_{2}(n)} \cdot c_{n}
$$

where

$$
\begin{aligned}
& F_{1}(n)=\left(2 n^{2}-2-k_{2}^{2} a^{2}\right)+2 k_{2} a j_{n+1}\left(k_{2} a\right) / j_{n}\left(k_{2} a\right) \\
& F_{2}(n)=(1-n)+k_{1} a j_{n+1}\left(k_{1} a\right)\left(j_{n}\left(k_{1} a\right)\right. \\
& F_{3}(n)=n-k_{1} a j_{n+1}\left(k_{1} a\right) / j_{n}\left(k_{1} a\right) .
\end{aligned}
$$

Note that the right hand side of equation (21) contains only the ratio of spherical Bessel functions. Using the series representation of $j_{n}(z)$, equation $(20)$, this ratio becomes which presents no computational difficulties. Similar comments apply to the other products of this form.

Recall from equation (18) that the complex argument of the spherical Bessel functions are $k_{1} r$ and $k_{2} r$, where $k_{1}$ and $k_{2}$ are given by equations (10) and (11). Since $\lambda^{*}$ and $\mu^{*}$ are complex functions of the frequency $\omega$, the same will be true of the wave speeds $k_{1}$ and $k_{2}$.

A general program for calculating spherical Bessel functions with complex arguments was not available and had to be written. It will be useful to have an idea of the value of these complex arguments. It has been suggested by Goldsmith (1966) that the mass density and bulk modulus of brain are the same as those for water, i.e. $\rho=1.94 \mathrm{slug} / \mathrm{ft}^{3}$ and $K^{*}=3 \times 10^{5}$ psi. The frequency used is $80 \mathrm{~Hz}$ or $502 \mathrm{rad} /$ sec. For a tried value of $\mu^{*}=(3+3 i)$ psi, then

$$
\lambda^{*}=K^{*}-\frac{2}{3} \mu^{*}=\left(3 \times 10^{5}-2\right)-2 i \mathrm{spi}
$$

and

$$
\begin{aligned}
& k_{1}^{2}=\left(0.0 .11-0.1418 \times 10^{-7} i\right) / \mathrm{ft}^{2} \\
& k_{2}{ }^{2}=(567-567 i) / \mathrm{ft}^{2} .
\end{aligned}
$$

The radius of monkey's head is measured as two in., so that

$$
\begin{aligned}
& k_{1} a=0.177 \times 10^{-1}-0.118 \times 10^{-6} i \\
& k_{2} a=4.3576-1.8046 i .
\end{aligned}
$$

With the above data and a shear modulus $\mu^{*}$ of this order of magnitude, the magnitude of the complex arguments will not be greater than 12. As is mentioned by Engin (1969), for arguments in this range, the series representation of equation (20) can be used to calculate $j_{n}(z)$ within a reasonable number of terms and a good accuracy. Otherwise, a recurrence

$$
\frac{j_{n}\left(k_{1} r\right)}{j_{n}\left(k_{1} a\right)}=\left(\frac{r}{a}\right)^{n} \frac{\left[1-\frac{k_{1}{ }^{2} r^{2} / 2}{1 !(2 n+3)}+\frac{\left(k_{1}{ }^{2} r^{2} / 2\right)^{2}}{2 !(2 n+3)(2 n+5)}-\cdots\right]}{\left[1-\frac{k_{1}{ }^{2} a^{2} / 2}{1 !(2 n+3)}+\frac{\left(k_{1}{ }^{2} a^{2} / 2\right)^{2}}{2 !(2 n+3)(2 n+5)}-\cdots\right]}
$$


formula or an asymptotic approximation for large arguments would be necessary. As was mentioned earlier, it is the ratio $j_{n}\left(k_{1} r\right) / j_{n}\left(k_{1} a\right)$ that is to be evaluated, not $j_{n}\left(k_{1} r\right)$ itself. This would depend on the series inside the brackets in equation (20). The above remarks show that it is reasonable to still use the series to find these ratios.

Recall that the arguments $k_{1} r$ and $k_{2} r$ contain the mass density $p$. In order to avoid a too small value of mass density, the dimension of $\rho$ was taken as slugs $/ \mathrm{ft}^{3}$. Since $k_{1} a$ and $k_{2} a$ are dimensionless, it means that radius $a$ has dimension of feet and $\mu^{*}$ will have dimensions of $\mathrm{lb} / \mathrm{ft}^{2}$. Finally, before calculating the stress or displacement or determining $\mu^{*}$, it is necessary to specify the maximum applied radial displacement $u_{0}$. From experimental measurement $u_{0}=4.3 \times 10^{-3} \mathrm{~cm}$ or $u_{0}=1.42 \times 10^{-4} \mathrm{ft}$. Since all the lengths have the dimension of feet, the calculated stress has dimension $\mathrm{lb} / \mathrm{ft}^{2}$.

In order to estimate the accuracy of calculation, $\sigma_{r r}$ at $r=2$ in or $\frac{1}{6} \mathrm{ft}$ and $\theta=0$ was evaluated. Since $P_{n}(\cos \theta)=1$ for $\theta=0$ and $P_{n}(\cos$ $\theta)<1$ for $\theta>0$, the series for $\sigma_{r r}(a, 0)$ should converge more slowly than if $\theta>0$. Any comments on the accuracy of calculations of $\sigma_{r r}(a, 0)$ should then apply for angle $\theta>0$. A plot of the real and imaginary $\sigma_{r r}(a, 0)$ vs. term $n$ shows slow oscillations which damp out as $n$ increases (see Wang, 1971).

The change in $\operatorname{Re}\left(\sigma_{r r}\right)$ in the first oscillation at $n=38$ is 14 per cent in the second oscillation at $n=113$ is 3 per cent and the last oscillation shown at $n=201$ is 1.5 per cent. $A$ similar comment holds for $I_{m}\left(\sigma_{r r}\right)$. Thus it appears reasonable to say that the infinite series has reached its limit value at 200 terms.

\section{Determination of complex shear modulus}

Having discussed details of the procedure for carrying out the various calculations, it is now possible to discuss the numerical determination of the complex moduli. Since it has been assumed that the bulk modulus is real valued for all frequencies, and its value is assumed known, the problem reduces to finding the complex shear modulus $\mu^{*}$ only.

For given geometry parameters, frequency, density and shear modulus $\mu^{*}$, it is now possible to calculate all the stress components in the sphere. However, the experimental data is in terms of the force under the probe which involves only the $\sigma_{r r}$ components through the relation

$$
F=\pi \delta^{2} \int_{0}^{\theta_{0}} \sigma_{r r}(a, \theta) \cos \theta \mathrm{d} \theta
$$

where $\delta$ is the probe radius and $\theta_{0}$ is angle of probe. Since the probe radius is small compared to the sphere radius, i.e. $\delta / a=\frac{1}{32}$, then it is reasonable to approximate this relation by

$$
F=\sigma_{r r}(a, 0) \times \text { probe area }
$$

or by $\sigma_{r r}(a, 0)=F /$ probe area.

By equation (18), the complex valued normal stress at $r=a$ is related to $\mu^{*}$ through the equation

$$
\begin{aligned}
& \sigma_{r r}(a, 0)=\frac{1}{a^{2}} \sum_{n=0}^{\infty} a_{n}\left\{\left[-\left(\lambda^{*}+2 \mu^{*}\right) k_{1}{ }^{2} a^{2}\right.\right. \\
& \left.\left.+2 \mu^{*}\left(n^{2}-n\right)\right] j_{n}\left(k_{1} a\right)+4 \mu^{*} k_{1} a j_{n+1}\left(k_{1} a\right)\right\} \\
& +\frac{2 \mu^{*}}{a^{2}} \sum_{n=1}^{\infty} \lambda_{n} b_{n}\left[(n-1) j_{n}\left(k_{2} a\right)-k_{2} a j_{n+1}\left(k_{2} a\right)\right]
\end{aligned}
$$

where $a_{n}, b_{n}$ and $k_{1}, k_{2}$ were defined in equations (19), (10) and (11) respectively. This defines a transcendental equation for $\mu^{*}$ in terms of known and experimental data.

Since the equation is so complicated, it was solved graphically. To this end, letting $\mu^{*}=$ $G^{\prime}+i G^{\prime \prime}$, plots were made of $\operatorname{Re}\left(\sigma_{r r}\right)$ vs. $G^{\prime}$ for various values of $G^{\prime \prime}$. A similar plot was made of $I m\left(\sigma_{r r}\right)$ vs. $G^{\prime \prime}$ for various values of $G^{\prime}$.

According to the results of experimental test 1 mentioned in Section 3, it was found that 
$\operatorname{Re}\left(\sigma_{r r}\right)=\frac{0.5775 \times 10^{3}}{0.1} \mathrm{dyne} / \mathrm{cm}^{2}$

$$
\cdot \cos \left(\tan ^{-1} 0.665\right)=10.05 \mathrm{lb} / \mathrm{ft}^{2}
$$

$\operatorname{Im}\left(\sigma_{r r}\right)=\frac{0.5775 \times 10^{3}}{0.1}$ dyne $/ \mathrm{cm}^{2}$

$$
\cdot \sin \left(\tan ^{-1} 0.665\right)=6.68 \mathrm{lb} / \mathrm{ft}^{2} .
$$

From the numerical results and this graphical representation, the following tables were constructed from $G^{\prime} . G^{\prime \prime}$ producing values of $\operatorname{Re}\left(\sigma_{r r}\right)$ and $I m\left(\sigma_{r r}\right)$ in the neighborhood of the experimental values.

\begin{tabular}{|c|c|c|c|}
\hline$G^{\prime \prime}$ & 2 & 3 & 4 \\
\hline 1 & 6.73 & $10 \cdot 82$ & 14.92 \\
\hline 2 & 6.79 & $10 \cdot 86$ & 14.96 \\
\hline 3 & 6.80 & $10 \cdot 87$ & 14.98 \\
\hline
\end{tabular}

Table 1. Values of $\operatorname{Re}\left(\sigma_{r r}\right)$

From Tables 1 and 2, it is seen that $\operatorname{Re}\left(\sigma_{r r}\right)$ varies strongly as $G^{\prime}$ varies. but is almost independent of $G^{\prime \prime}$. Similarly. $\operatorname{Im}\left(\sigma_{r r}\right)$ depends strongly on $G^{\prime \prime}$, but only slightly on $G^{\prime}$. Hence a plot of $\operatorname{Re}\left(\sigma_{r r}\right)$ vs. $G^{\prime}$ for different $G^{\prime \prime}$ yields only one curve which is shown in Fig. 3. There is also only one curve for $\operatorname{Im}\left(\sigma_{r r}\right)$ vs. $G^{\prime \prime}$ for different $G^{\prime}$ as shown in Fig. 4. Al-

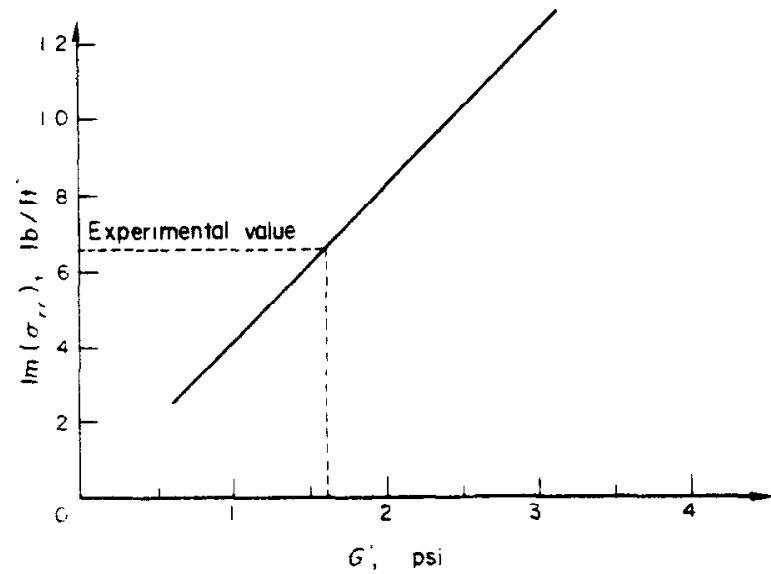

Fig. 4. Imaginary part of normal stress vs. imaginary part of complex shear modulus. though $\operatorname{Re}\left(\sigma_{r r}\right)$ is almost independent of $G^{\prime \prime}$. it is very sensitive to $G^{\prime}$. Thus it is possible to get a quite accurate value for $G^{\prime}$ from $R e$ $\left(\sigma_{r r}\right)$, and an accurate value of $G^{\prime \prime}$ from $I m$ $\left(\sigma_{r r}\right)$. From Fig. 3, it is found that $G^{\prime}=2.83$ best fits the experimental value while from Fig. 4 , it is found that $G^{\prime \prime}=1.62$ is the best choice. To test the accuracy of this graphical determination, $\mu^{*}=2.83+1.62 i$ was used in a calculation of $\sigma_{r r}$. It was found that $\sigma_{r r}=$ $(10.07+6.62 i) \mathrm{b} / \mathrm{ft}^{2}$ which differs from the experimental data by 1 per cent. This is within the accuracy of the calculation. Thus the complex shear modulus of monkey brain at a frequency of $80 \mathrm{~Hz}$ is $\mu^{*}=(2.83+1.62 i)$ psi. About $150 \mathrm{sec}$ computation time (by IBM 360 Model EC 170) were necessary to get enough data points so as to find the value of $\mu^{*}$ corresponding to a given frequency $\omega$. This does not include the time for compiling the program and determining the coefficients of the series of Legendre polynomial which represents the given surface radial displacement. $u(\theta)$.

\begin{tabular}{|c|c|c|c|}
\hline ; & 2 & 3 & 4 \\
\hline 1 & $4 \cdot 12$ & $4 \cdot 10$ & 4.08 \\
\hline 2 & $8 \cdot 13$ & $8 \cdot 15$ & $8 \cdot 16$ \\
\hline 3 & $12 \cdot 24$ & $12 \cdot 22$ & $12 \cdot 23$ \\
\hline
\end{tabular}

Table 2. Values of $\operatorname{Im}\left(\sigma_{r r}\right)$

If the frequency or any other geometric parameter differs from the above used value, $R e\left(\sigma_{r r}\right)$ and $\operatorname{lm}\left(\sigma_{r r}\right)$ may vary with both $G^{\prime}$ and $G^{\prime \prime}$. Then the procedure for finding the appropriate value of the complex shear modulus $\mu^{*}$ by a graphical method becomes more complicated and will be outlined as follows. First. $R e\left(\sigma_{r r}\right)$ vs. $G^{\prime}$ for different $G^{\prime \prime}$ is plotted as shown in Fig. 5, for example. Since $R e\left(\sigma_{r r}\right)$ may depend on both $G^{\prime \prime}$, this produces a family of curves.

From this plot, one can find a set of points $P_{1}, P_{2} . P_{3} \ldots$. The coordinates of these points are values of the complex shear modulus 


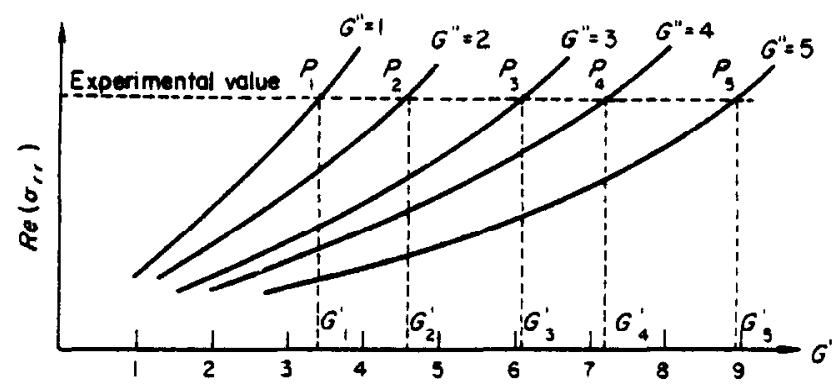

Fig. 5. $\operatorname{Re}\left(\sigma_{\pi}\right)$ vs. $G^{\prime}$ with various $G^{\prime \prime}$ for $\operatorname{Re}\left(\sigma_{r r}\right)$ dependent on $G^{\prime \prime}$.

which match the experimental result, i.e. $P_{1}\left(G_{1}^{\prime}+i\right), P_{2}\left(G_{2}^{\prime}+2 i\right), P_{3}\left(G_{3}^{\prime}+3 i\right), \ldots$ Next, these values of $\mu^{*}$ are then used to calculate I $m\left(\sigma_{r r}\right)$ and plot a curve of $\operatorname{Im}\left(\sigma_{m}\right)$ vs. $G^{\prime \prime}$. The curve might look like that in Fig. 6.

From this curve, one can find a uniquely defined point corresponding to the same value of $\operatorname{Im}\left(\sigma_{r r}\right)$ as the experimental value. For example, in Fig. $6, G^{\prime \prime}=4.6$ will be the desired value. In determining $G^{\prime}$, an interpolation method is used between $G_{4}^{\prime}$ and $G_{5}^{\prime}$. This procedure locates the range of values of $\mu^{*}$ corresponding to experimental data. Once this has been done, additional points can be plotted so as to obtain $\mu^{*}$ to the desired accuracy.

Stress and displacement field produced by the probe test

The probe test produces a stress and displacement field throughout the sphere. It is of interest to examine the nature of distribution of these fields. The stress and displacement fields produced by the probe test, within the context of the modulus of this test presented here, can be obtained by substituting the experimentally determined $\mu^{*}$ back into equation (18). These are plotted in Figs. 7-10.

Figure 7 shows the amplitude of radial stress $\left|\sigma_{\pi}\right|$ as a function of the azimuth angle $\theta$. It is easily seen that $\left|\sigma_{r r}\right|$ decreases rapidly as $\theta$ increases. At about $7^{\circ}$, for all radii, $\left|\sigma_{\pi}\right|$ is less than 10 per cent of its maximum value. Around $10^{\circ},\left|\sigma_{r}\right|$ increases slightly due to the restraining influence of the boundary of the hole in the spherical shell. For $\theta>15^{\circ},\left|\sigma_{r r}\right|$ is at most 3 per cent of the maximum stress, for all radii. Figure 7 also shows that $\left|\sigma_{r r}\right|$ decreases rapidly as $r$ approaches the center of the sphere. At $r=0.8 a,\left|\sigma_{r r}\right|$ has already reduced by 85 per cent of its maximum value. For $r<0.6 a,\left|\sigma_{r r}\right|$ is less than 2 per cent of maximum $\left|\sigma_{r r}\right|$.

The distribution of $\left|\sigma_{\theta \theta}\right|$ and $\left|\sigma_{\varphi \phi}\right|$ with respect to $r$ and $\theta$ is much the same as that of

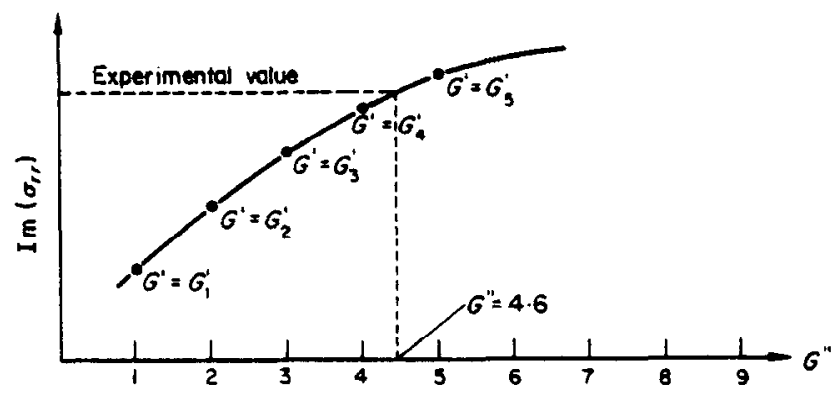

Fig. 6. $\operatorname{Im}\left(\sigma_{r r}\right)$ vs. $G^{\prime \prime}$ with some particular values of 


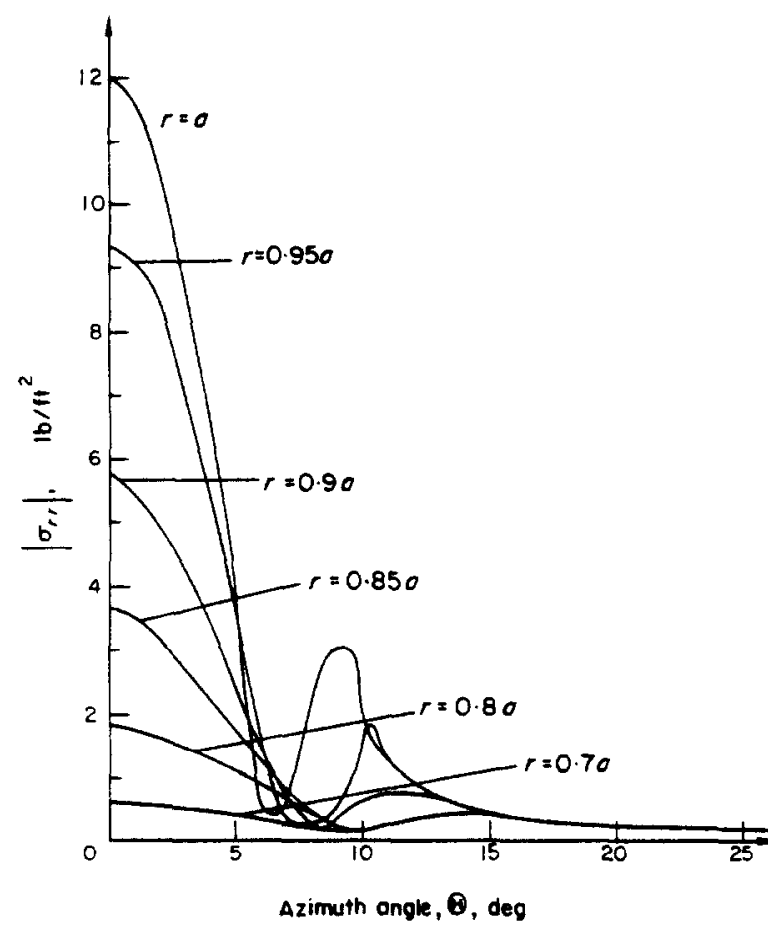

Fig. 7. $\left|\sigma_{r r}\right| v s$. angle $\theta$ with various radii $r$.

$|\sigma|_{r r}$ and is not shown for reasons of brevity. Their graphs can be found in the work by Wang (1971).

The behavior of the shear stress $\left|\sigma_{r \theta}\right|$ is shown in Fig. 8. Its maximum value occurs

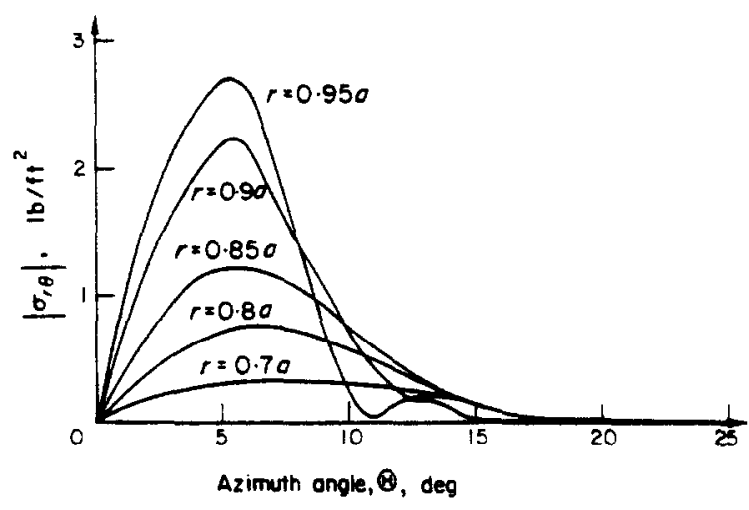

Fig. 8. $\left|\sigma_{r \theta}\right|$ us. angle $\theta$ with various radii $r$.

around $r=0.95 a$ and $\theta=5^{\circ}$, and is seen to be about one quarter to the maximum $\left|\sigma_{r r}\right|$. Thus, even though the bulk modulus is large compared to $\mu^{*}$. significant shear stresses are induced by the probe test. Shear stress $\left|\sigma_{r \theta}\right|$ decreases as $\theta$ increases and radius decreases so that for $r \leqslant 0.6 a$ and $\theta \geqslant 15^{\circ}$, the shear has decreased 95 per cent of its maximum value.

Figures 9 and 10 show the displacement component vs. angle $\theta$ for different radii. From Fig. 9, it is seen that near the surface. the radial displacement $u_{r}$ has its largest values for $r \geqslant 0.8 a$ and $\theta<10^{\circ}$. Figure 10 shows that the tangential displacement $u_{\theta}$ has its maximum value near the surface and between $\theta=5^{\circ}$ and

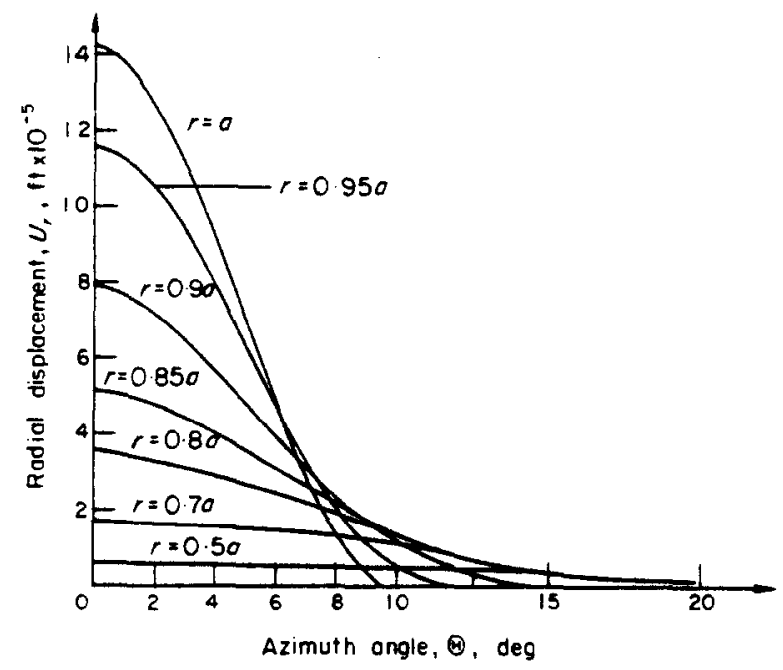

Fig. 9. Normal displacement $u_{r} v s . \theta$ with various radius 


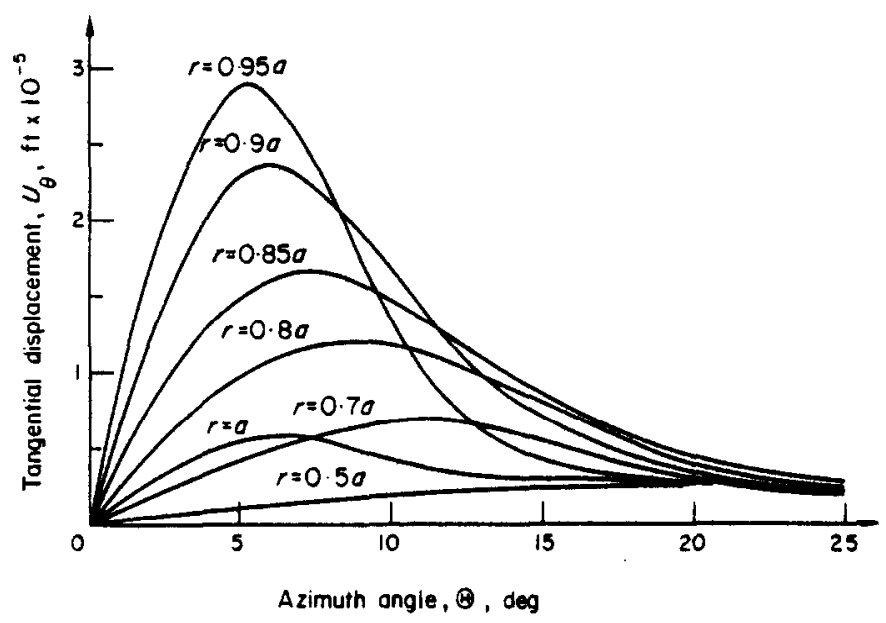

Fig. 10. Meridanal displacement $u_{\theta}$ vs. $\theta$ with various radius $r$.

$7^{\circ}$. Its value decreases rapidly with radius. Also, it shows that on the surface, $r=a$, the maximum displacement $u_{\theta}$ has only 3 per cent of the maximum value of $u_{r}$. This means that $u_{\theta}$ is relatively small compared with the radial displacement $u_{r}$. Then the shear force between the sphere and container due to the viscosity of the fluid will be very small. So the assumption of zero shear stress on the surface is quite reasonable.

These graphs show that the most significant value of the stress and displacement components occur in the region for which $r \geqslant 0.8 a$ and $\theta \leqslant 15^{\circ}$. This implies that the influence of the probe is confined to a small region around the hole in the spherical case.

\section{FINAL COMMENTS}

Because the bulk modulus $k^{*}$ is large compared to the shear modulus $\mu^{*}$, it is reasonable to expect that the probe test would induce a small shear stress compared to normal stress and possibly be a poor test for determining $\mu^{*}$. The results presented here suggest that, to the contrary, the probe test appears to be quite adequate for measuring $\mu^{*}$. The Tables 1 and 2 and Figs. 3 and 4 show that the force under the probe, calculated by equation (22) is sensitive to change in $\mu^{*}$, and that $\mu^{*}$ can be readily determined. Secondly, as mentioned earlier, the maximum $\left|\sigma_{r \theta}\right|$ is about one quarter of maximum $\left|\sigma_{r r}\right|$. Thus, the probe test does indeed induce reasonable shear stress and can be considered a meaningful method for obtaining $\mu^{*}$.

It is possible to convert the complex shear modulus $\mu^{*}$ to the complex tensile modulus $E^{*}$. by means of equation (1). Letting $\sigma_{11}$ be the only nonzero stress component in equation (1), one obtains

$$
\sigma_{11}=E^{*}(i \omega) \epsilon_{11}
$$

where

$$
E^{*}=3 \mu^{*} /\left(1+\frac{\mu^{*}}{3 k^{*}}\right)
$$

As mentioned earlier $\left|k^{*}\right|>\left|\mu^{*}\right|$ in brain tissue, so that

$$
E^{*} \simeq 3 \mu^{*}
$$

Hence the complex tensile modulus $E^{*}$ of monkey's brain at a frequency of $80 \mathrm{~Hz}$ has the value

$$
E^{*}=(8 \cdot 5+4 \cdot 9 i) \text { psi. }
$$


Galford and McElhaney (1970) published a value of complex tensile modulus of monkey's brain by running a free vibration test. By using the approximate relation between complex tensile modulus and shear modulus of brain, $E^{*}=3 \mu^{*}$, the amplitude of their value is different from that reported here by a factor of $1.5 \sim 2$. Since the amount of data is limited. it is difficult to make other comparisons. Possible reasons for the different values are stated as follows:

1. Galford and McElhaney performed tests in vitro. The data utilized here was obtained in vivo. This alone should account for much of the difference.

2. Brain tissue is not homogeneous. Specimens taken from different locations will have different material properties. In this investigation, the material property which is found numerical and graphical methods is the effective material property of whole brain.

3. The assumed boundary conditions are not exactly the same as in the real test.

4. It is possible that material is non-linear and also that the relative large preset causes geometric nonlinearity.

Acknowledgement - This investigation was supported by the Biomechanics Department of the Highway Safety Research Institute of The University of Michigan under contract No. Ph-43-67-1136 sponsored by the National Institute of Neurological Diseases and Stroke.

\section{REFERENCES}

Bland. D. R. (1960). The Theory of Linear Viscoelasticity. p. 67. Pergamon Press. Oxford.

Engin. A. E. (1969). The axisymmetric response of a fiuidfilled spherical shell to a local radial impulse. A model for head injury. J. Biomechanics 2. 325-341.

Engin. A. E. and Wang. H. C. (1970), A mathematical model to determine viscoelastic behavior of in vivo primate brain. J. Biomechanics 3, 283-296.

Fallenstein. G. T.. Hulce. V. D. and Melvin. J. W. (1969), Dynamic mechanical properties of human brain tissue. J. Biomechanics 2. 217-226.

Ferry. 1. D. (1956). Physik der hochpolymeren 4. Chapter 1. Springer. Berlin.

Flugge, W, (1967). Viscoelasticity. Blaisdel, Waltham. Massachusetts.

Galford. J. E. and McElharney. J. H. (1970), Some viscoelastic properties of scalp. brain and dura. J. Biomechanics 3.211.
Goldsmith. W. (1966). The physical processes producing head injuries. In Head Injury, (Edited by W. F. Caveness and A. E. Walker) 358 Lippincott, Philadelphia.

Gross. B. (1953), Mathematical Structure of the Theories of Viscoelasticity. Hermann, G. C. Editeurs. Paris.

Gurtin. M. E. and Stemberg. E. (1962). On the linear theory of viscoelasticity. Arch. Ration. Mech. Analysis 11, 291-356.

Handbook of Mathematical Functions and Formulas. Graphs and Mathematical Tables. (1964) Edited by Milton Abramowitz and Irene A. Stegan) National Bureau of Standards. AM 555.

Holbourn, A. H. S. (1943), Lancet 2, 438-441.

Jamison, C. E. et al. (1968), Viscoelastic properties of soft tissue by discrete model characterization. J. Biomechanics 1. 35-46.

Love, A. E. H. (1944). A Treatise on the Mathematical Theory of Elasticity. 47, Dover. New York.

Meivin, J. W. (1970). Personal File. Highway Safety Research Institute, the University of Michigan. Ann Arbor, Michigan.

Robbins, D. H. and Roberts, V. L. (1967), Six Month Main Report on Contract No. PH-43-67-1136, 15.

Sokoinikoff, 1. S. (1956), Mathematical Theory of Elasticity. 367-371, McGraw-Hill, New York.

Wang, Han Chou, (1971) The response of viscoelastic solid sphere to a local excitation: A theoretical model to determine mechanical properties of in vivo primate brain, doctoral dissertation. The University of Michigan.

\begin{tabular}{|c|c|}
\hline \multicolumn{2}{|c|}{ NOMENCLATURE } \\
\hline$\delta_{i j}$ & Kronecker delta \\
\hline$\epsilon_{i j}$ & Strain tensor \\
\hline$\sigma_{i}$ & Stress tensor \\
\hline$t$ & Time \\
\hline$\omega$ & Angular frequency \\
\hline$\mu(t) \cdot k(t)$ & $\begin{array}{l}\text { Relaxation function governing } \\
\text { shear and volumetric dilatation } \\
\text { response. respectively }\end{array}$ \\
\hline$\mu^{*}(i \omega), \lambda^{*}(i \omega), k^{*}(i \omega)$ & $\begin{array}{l}\text { Complex shear, bulk and Lamé } \\
\text { moduli }\end{array}$ \\
\hline$E^{*}$ & Complex tensile modulus \\
\hline$a$ & Radius of solid viscoelastic sphere \\
\hline$r, \theta, \varphi$ & Spherical coordinates \\
\hline $\mathbf{u}$ & $\begin{array}{l}\text { Displacement vector in space } \\
\text { coordinates only }\end{array}$ \\
\hline $\mathbf{X}$ & Space vector \\
\hline$u_{r} \cdot u_{\theta} \cdot u_{\psi}$ & $\begin{array}{l}\text { Components of displacement vec- } \\
\text { tor in spherical coordinates }\end{array}$ \\
\hline$\rho$ & Mass density \\
\hline$\Phi$ & Scalar displacement potential \\
\hline$\Psi$ & Vector displacement potential \\
\hline$a_{n} \cdot b_{n}$ & $\begin{array}{l}\text { Coefficients of infinite series ex- } \\
\text { pansions of displacements } \\
\text { potentials } \Phi \text { and } \Psi \text {. respectively }\end{array}$ \\
\hline$c_{n}$ & $\begin{array}{l}\text { Coefficient of the Legendre poly- } \\
\text { nomial expansion of the local } \\
\text { applied radial displacement }\end{array}$ \\
\hline$j_{n}(z), y_{n}(z)$ & $\begin{array}{l}\text { Spherical Bessel function of first } \\
\text { and second kind. respectively }\end{array}$ \\
\hline
\end{tabular}

NOMENCLATURE

Strain tensor

$\mu(t), k(t)$ Relaxation function governing shear and volumetric dilatation response. respectively modul

Complex tensile modulus

Spherical coordinates

u Displacement vector in space coordinates only

$u_{r} . u_{\theta} . u_{4}$ Components of displacement vector in spherical coordinates

$\rho$ Mass density

$\Phi$ Scalar displacement potentia

$\boldsymbol{\Psi}$ Vector displacement potential pansions of displacements potentials $\Phi$ and $\boldsymbol{\Psi}$. respectively applied radial displacemen and second kind. respectively nomial expansion of the local 
$P_{n}(x), P_{n}{ }^{1}(x)$ Legendre and Associate Legendre Polynomials of first kind

Im ( ). $\operatorname{Re}$ ( ) Imaginary and real part of complex function inside the parentheses, respectively $\nabla \quad$ Gradient operator

$\nabla$. Divergence operator

$\nabla x$ Curl operator

$\nabla^{2}$ Laplacian 